\title{
On complete monotonicity of the Riemann zeta function
}

Ruiming Zhang*

${ }^{*}$ Correspondence:
ruimingzhang@gmail.com
College of Science, Northwest A\&F
University, Yangling, Shaanxi
712100, P.R. China

"Correspondence:

College of Science, Northwest A\&F

712100, P.R. China

\begin{abstract}
Under the assumption of the Riemann hypothesis for the Riemann zeta function and some Dirichlet $L$-series we demonstrate that certain products of the corresponding zeta functions are completely monotonic. This may provide a method to disprove a certain Riemann hypothesis numerically.
\end{abstract}

MSC: Primary 30E15; secondary 33D45

Keywords: Riemann zeta function; Dirichlet series; Riemann hypothesis; complete monotonic functions

\section{Introduction}

The Riemann zeta function $\zeta(s)$ can be defined by

$$
\zeta(s)=\sum_{n=1}^{\infty} \frac{1}{n^{s}}, \quad \Re(s)>1,
$$

and on the rest of the complex plane by analytic continuation. It is known that the extended $\zeta(s)$ is meromorphic with infinitely many zeros at $-2 n$ for $n \in \mathbb{N}$ (a.k.a trivial zeros) and with infinitely many zeros within the vertical strip $0<\Re(s)<1$ (nontrivial zeros). The Riemann hypothesis for $\zeta(s)$ says that all nontrivial zeros are actually on the critical line $\Re(s)=\frac{1}{2}$.

For any complex number $z \in \mathbb{C}$, let $\Gamma(z)$ be Euler's Gamma function defined by [1-8]

$$
\frac{1}{\Gamma(z)}=z \prod_{j=1}^{\infty}\left(1+\frac{z}{j}\right)\left(1+\frac{1}{j}\right)^{-z} .
$$

Then, the Riemann $\Xi(z)$ function $[1-7]$

$$
\Xi(z)=-\frac{1+4 z^{2}}{8} \pi^{-\frac{1+2 i z}{4}} \Gamma\left(\frac{1+2 i z}{4}\right) \zeta\left(\frac{1+2 i z}{2}\right)
$$

is an even entire function of order 1 . The celebrated Riemann hypothesis is equivalent to the statement that $\Xi(z)$ has only real zeros.

\section{Springer}

O2014 Zhang; licensee Springer. This is an Open Access article distributed under the terms of the Creative Commons Attribution License (http://creativecommons.org/licenses/by/2.0), which permits unrestricted use, distribution, and reproduction in any medium, provided the original work is properly cited. 
Let $\chi(n)$ be a real primitive character with modulus $m$; the function $L(s, \chi)$ is defined by $[3,8]$

$$
L(s, \chi)=\sum_{n=1}^{\infty} \frac{\chi(n)}{n^{s}}, \quad \Re(s)>1
$$

Let

$$
\alpha= \begin{cases}0, & \chi(-1)=1 \\ 1, & \chi(-1)=-1\end{cases}
$$

then

$$
\Xi(z, \chi)=\left(\frac{\pi}{m}\right)^{-(1+2 \alpha+2 i z) / 4} \Gamma\left(\frac{1+2 \alpha+2 i z}{4}\right) L\left(\frac{1+2 i z}{2}, \chi\right)
$$

is an even entire function of order 1 . The Riemann hypothesis for $L(s, \chi)$ is equivalent to $\Xi(z, \chi)$ having only real zeros.

Given real numbers $a, b$ with $a<b$ and an indefinite differentiable real valued function $f(x)$ on $(a, b), f(x)$ is called completely monotonic on $(a, b)$ if $(-1)^{m} f^{(m)}(x) \geq 0$ for all $x \in(a, b)$ and $m=0,1, \ldots$ In this work, under the assumptions of the Riemann hypothesis for the Riemann zeta function and certain $L$-series, we apply the ideas from [8,9] to prove that some products of these zeta functions are completely monotonic. This complete monotonicity may provide a method to disprove a certain Riemann hypothesis via numerical methods.

\section{Main results}

Lemma 1 Given a non-increasing sequence of positive numbers such that

$$
\sum_{n=1}^{\infty}\left|\lambda_{n}\right|<\infty
$$

then, the entire function

$$
f(x)=\prod_{n=1}^{\infty}\left(1-x \lambda_{n}\right)
$$

is completely monotonic on $\left(-\infty, \lambda_{1}^{-1}\right)$.

Proof It is a direct consequence of Theorem 1 of [8].

Assuming the Riemann hypothesis is true, we list all positive zeros of $\Xi(z)$ as

$$
z_{1} \leq z_{2} \leq \cdots \leq z_{n} \leq \cdots
$$

and $z_{1}$ is approximately 14.1347 . Then,

$$
\Xi(z)=\Xi(0) \prod_{n=1}^{\infty}\left(1-\frac{z^{2}}{z_{n}^{2}}\right)
$$


Thus,

$$
\begin{aligned}
& \prod_{n=1}^{\infty}\left(1-\frac{z}{z_{n}^{2}}\right)=\frac{\Xi(\sqrt{z})}{\Xi(0)}, \\
& \frac{\Xi\left(z^{\frac{1}{4}}\right) \Xi\left(i z^{\frac{1}{4}}\right)}{\Xi^{2}(0)}=\prod_{n=1}^{\infty}\left(1-\frac{z}{z_{n}^{4}}\right),
\end{aligned}
$$

and

$$
\frac{\Xi\left(z^{\frac{1}{6}}\right) \Xi\left(\rho z^{\frac{1}{6}}\right) \Xi\left(\rho^{2} z^{\frac{1}{6}}\right)}{\Xi^{3}(0)}=\prod_{n=1}^{\infty}\left(1-\frac{z}{z_{n}^{6}}\right)
$$

for $0 \leq \arg (z)<2 \pi$, where $\rho=e^{\frac{2 \pi i}{3}}$. In fact, for any positive integer $\ell>1$ and assume that $\rho_{\ell}$ is a primitive $\ell$ th root of unity; then we have

$$
\frac{\prod_{j=1}^{\ell} \Xi\left(\rho_{\ell}^{j} z^{\frac{1}{2 \ell}}\right)}{\Xi^{\ell}(0)}=\prod_{n=1}^{\infty}\left(1-\frac{z}{z_{n}^{2 \ell}}\right) .
$$

Corollary 2 Under the Riemann hypothesis, let $z_{1}$ be the least positive zeros of $\Xi(z)$; then the function $\Xi(\sqrt{z})$ is completely monotonic for $z \in\left(-\infty, z_{1}^{2}\right), \Xi\left(z^{\frac{1}{4}}\right) \Xi\left(i z^{\frac{1}{4}}\right)$ is completely monotonic for $z \in\left(-\infty, z_{1}^{4}\right)$, and $\Xi\left(z^{\frac{1}{6}}\right) \Xi\left(\rho z^{\frac{1}{6}}\right) \Xi\left(\rho^{2} z^{\frac{1}{6}}\right)$ is completely monotonic for $z \in\left(-\infty, z_{1}^{6}\right)$. Let $\rho_{\ell}$ be a primitive $\ell$ th root of unity for some positive integer $\ell$; then $\prod_{j=1}^{\ell} \Xi\left(\rho_{\ell}^{j} z^{\frac{1}{2 \ell}}\right)$ is completely monotonic for $z \in\left(-\infty, z_{1}^{2 \ell}\right)$.

Proof Notice that $\Xi(0)$ is a positive constant, and the claims are obtained by applying Corollary 1 to equations (2.5)-(2.8).

Assuming the Riemann hypothesis for $L(s, \chi)$, we list all the positive zeros for $\Xi(z, \chi)$ as [8]

$$
z_{1}(\chi) \leq z_{2}(\chi) \leq \cdots \leq z_{n}(\chi) \leq \cdots .
$$

Then

$$
\Xi(z, \chi)=\Xi(0, \chi) \prod_{n=1}^{\infty}\left(1-\frac{z^{2}}{z_{n}(\chi)^{2}}\right) .
$$

Evidently,

$$
\Xi(0, \chi) \neq 0,
$$

otherwise $\Xi(z, \chi) \equiv 0$, which is clearly false. Thus,

$$
\prod_{n=1}^{\infty}\left(1-\frac{z}{z_{n}(\chi)^{2}}\right)=\frac{\Xi(\sqrt{z}, \chi)}{\Xi(0, \chi)}
$$


for $0 \leq \arg (z)<2 \pi$. Furthermore,

$$
\frac{\Xi\left(z^{\frac{1}{4}}, \chi\right) \Xi\left(i z^{\frac{1}{4}}, \chi\right)}{\Xi^{2}(0)}=\prod_{n=1}^{\infty}\left(1-\frac{z}{z_{n}^{4}(\chi)}\right)
$$

and

$$
\frac{\Xi\left(z^{\frac{1}{6}}, \chi\right) \Xi\left(\rho z^{\frac{1}{6}}, \chi\right) \Xi\left(\rho^{2} z^{\frac{1}{6}}, \chi\right)}{\Xi^{3}(0)}=\prod_{n=1}^{\infty}\left(1-\frac{z}{z_{n}^{6}(\chi)}\right)
$$

for $0 \leq \arg (z)<2 \pi$, where $\rho=e^{\frac{2 \pi i}{3}}$. Let $\rho_{\ell}$ be a primitive $\ell$ th root of unity for some positive integer $\ell$; then we have

$$
\frac{\prod_{j=1}^{\ell} \Xi\left(\rho_{\ell}^{j} z^{\frac{1}{2 \ell}}, \chi\right)}{\Xi^{\ell}(0, \chi)}=\prod_{n=1}^{\infty}\left(1-\frac{z}{z_{n}^{2 \ell}(\chi)}\right)
$$

Corollary 3 Assume that the Riemann hypothesis is true for $L(s, \chi)$ and $z_{1}(\chi)$ is the least positive zero of $\Xi(z, \chi)$; then the function $\frac{\Xi(\sqrt{z}, \chi)}{\Xi(0, \chi)}$ is completely monotonic for $z \in\left(-\infty, z_{1}^{2}(\chi)\right)$, $\frac{\Xi\left(z^{\frac{1}{4}}, \chi\right) \Xi\left(i z^{\frac{1}{4}}, \chi\right)}{\Xi^{2}(0)}$ is completely monotonic for $z \in\left(-\infty, z_{1}^{4}(\chi)\right)$, and $\frac{\Xi\left(z^{\frac{1}{6}}, \chi\right) \Xi\left(\rho z^{\frac{1}{6}}, \chi\right) \Xi\left(\rho^{2} z^{\frac{1}{6}}, \chi\right)}{\Xi^{3}(0)}$ is completely monotonic for $z \in\left(-\infty, z_{1}^{6}(\chi)\right)$. Let $\rho_{\ell}$ be a primitive eth root of unity for some positive integer $\ell$, then $\prod_{j=1}^{\ell} \Xi\left(\rho_{\ell}^{j} z^{\frac{1}{2 \ell}}, \chi\right)$ is completely monotonic for $z \in\left(-\infty, z_{1}^{2 \ell}(\chi)\right)$.

Proof These are consequences of Lemma 1 and equations (2.12)-(2.15).

\section{Competing interests}

The author declares that they have no competing interests.

\section{Acknowledgements}

This research is partially supported by National Natural Science Foundation of China, grant No. 11371294

Received: 29 September 2013 Accepted: 13 December 2013 Published: 09 Jan 2014

\section{References}

1. Abramowitz, M, Stegun, IA (eds.): Handbook of Mathematical Functions with Formulas, Graphs, and Mathematical Tables, Tenth printing with corrections. Natl. Bur. of Standards, New York (1972)

2. Andrews, GE, Askey, R, Roy, R: Special Functions. Cambridge University Press, Cambridge (1999)

3. Davenport, H: Multiplicative Number Theory. Springer, New York (2000)

4. Erdélyi, A: Higher Transcendental Functions, Vol. I. Krieger, Malabar (1985)

5. Erdélyi, A: Higher Transcendental Functions, Vol. II. Krieger, Malabar (1985)

6. Erdélyi, A: Higher Transcendental Functions, Vol. III. Krieger, Malabar (1985)

7. Titchmarsh, EC: The Theory of Riemann Zeta Function, 2nd edn. Clarendon Press, New York (1987)

8. Zhang, R: Sums of zeros for certain special functions. Integral Transforms Spec. Funct. 21(5), 351-365 (2010)

9. Ismail, MEH, Zhang, R: Completely monotonic Fredholm determinants. J. Approx. Theory (submitted) 